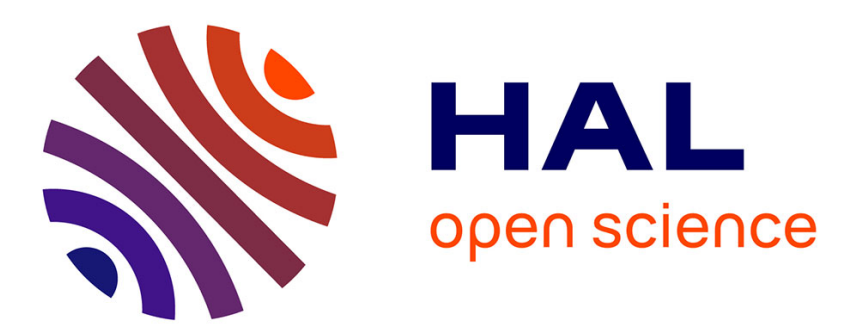

\title{
Rôle des interfaces dans les mécanismes élémentaires de déformation des superalliages base nickel
}

\author{
B. Décamps, V. Brien, J. Pénisson, A. Morton, M. Condat
}

\section{To cite this version:}

B. Décamps, V. Brien, J. Pénisson, A. Morton, M. Condat. Rôle des interfaces dans les mécanismes élémentaires de déformation des superalliages base nickel. Journal de Physique IV Proceedings, 1996, 06 (C2), pp.C2-263-C2-268. 10.1051/jp4:1996238 . jpa-00254216

\section{HAL Id: jpa-00254216 https://hal.science/jpa-00254216}

Submitted on 1 Jan 1996

HAL is a multi-disciplinary open access archive for the deposit and dissemination of scientific research documents, whether they are published or not. The documents may come from teaching and research institutions in France or abroad, or from public or private research centers.
L'archive ouverte pluridisciplinaire HAL, est destinée au dépôt et à la diffusion de documents scientifiques de niveau recherche, publiés ou non, émanant des établissements d'enseignement et de recherche français ou étrangers, des laboratoires publics ou privés. 


\title{
Rôle des interfaces dans les mécanismes élémentaires de déformation des superalliages base nickel
}

\author{
B. Décamps, V. Brien*, J.M. Pénisson**, A.J. Morton*** et M. Condat \\ Laboratoire de Métallurgie Structurale, URA 1107 du CNRS, Université Paris-Sud, Bât. 413, 91405 Orsay \\ cedex, France \\ * Laboratoire d'Etudes des Microstructures, UMR 104, BP. 72, 92322 Châtillon s/Bagneux, France \\ ** CEADépartement de Recherche Fondamentale sur la Matière Condensée (SP2M/S), CENG, 17 Rue des \\ Martyrs, 38054 Grenoble Cedex 9, France \\ *** CSIRO, Division of Materials Science and Technology, Private Bag 33, Rosebank MDC, Clayton, \\ Victoria 3169, Australie
}

\begin{abstract}
Ni-based superalloys used in the aeronautic industry. In particular, the description of the interaction between dislocations and interfaces is essential to understand the behaviour of these alloys under thermal and/or mechanical loading. A fine analysis at different levels of the behaviour of these dislocations after different mechanical tests (creep, dynamical tests, fatigue) allowed us to describe the elementary mechanisms that control the deformation.
\end{abstract}

\section{INTRODUCTION}

Les superalliages base nickel sont beaucoup utilisés dans l'industrie aéronautique en raison de leurs excellentes propriétés mécaniques à chaud. Ce sont des alliages biphasés (matrice CFC et précipités ordonnés $\gamma^{\prime}$ de structure $\mathrm{L}_{2}$ ). Il est bien connu que la précipitation $\gamma^{\prime}$ est responsable de ces caractéristiques mécaniques, les interfaces servant d'obstacle à la déformation.

L'objectif de cet article est de montrer comment l'étude fine, notamment aux interfaces, des microstructures résultant de différents types de déformation (fluage ; essais dynamiques ; fatigue oligocyclique) nous a permis de remonter aux mécanismes élémentaires régissant la déformation. Dans certains cas, il a pu être établi des corrélations entre le comportement à l'échelle de la microstructure et les propriétés macroscopiques.

\section{MATERIAUX ET PROCEDURE EXPERIMENTALE}

Des essais mécaniques ont été effectués sur des monocristaux de superalliages base nickel appelés AM1 (composition : $\mathrm{Ni}-6,5 \mathrm{Co} ; 7,5 \mathrm{Cr} ; 5,3 \mathrm{Al} ; 5,5 \mathrm{~W} ; 8 \mathrm{Ta} ; 2 \mathrm{Mo} ; 1,2 \mathrm{Ti}$ ) et $\mathrm{AM} 3$ (composition : $\mathrm{Ni}-5,5 \mathrm{Co}$; $8 \mathrm{Cr} ; 6 \mathrm{Al} ; 5 \mathrm{~W} ; 3,5 \mathrm{Ta} ; 2,25 \mathrm{Mo} ; 2 \mathrm{Ti})$. Après un traitement thermique de mise en solution et précipitation, les précipités $\gamma$, de structure $\mathrm{L}_{2}$, ont une fraction volumique voisine de $70 \%$ et sont cubiques (taille de l'ordre de $450 \mathrm{~nm})$. L'écart paramétrique $\delta\left(2\left(a \gamma^{\prime}-a \gamma\right) /\left(a \gamma^{\prime}+a \gamma\right)\right)$ est égal à $-10^{-3}$ à $950^{\circ} \mathrm{C}$.

Les essais mécaniques effectués suivant l'axe $<001>$ sont les suivants :

- fluage traction sur l'AM3 à $760^{\circ} \mathrm{C}$ sous $750 \mathrm{MPa}$, arrêté à $0,49 \%$ de déformation plastique.

- fluage compression sur l'AM3 à $810^{\circ} \mathrm{C}$ sous $650 \mathrm{MPa}$, arrêté à $0,4 \%$ de déformation plastique.

- compression dynamique sur l'AM1 à $950^{\circ} \mathrm{C}$, arrêtée à $0,4 \%$ et $0,6 \%$ (pour les observations par microscopie electronique en haute résolution) de déformation plastique.

- traction dynamique sur l'AM1 à $950^{\circ} \mathrm{C}$, arrêtée à $1,4 \%$ de déformation plastique.

- fatigue oligocyclique sur l'AM1 à $950^{\circ} \mathrm{C}$, avec $R \varepsilon=0\left(R \varepsilon=\varepsilon_{\min } / \varepsilon_{\max }\right)$ et $\mathrm{f}=0,25 \mathrm{~Hz}$ pour différentes valeurs de déformation totale imposée et un nombre de cycles variable.

Les observations sont faites à l'aide des microscopes Jeol 2000EX et Jeol $200 \mathrm{CX}$ en utilisant les techniques du champ faible [1] et du champ clair et d'un microscope Jeol 4000EX pour la haute résolution (les images 
sont prises à $600 \mathrm{~K}$ dans des conditions d'épaisseur et de défocalisation où les colonnes atomiques sont blanches).

\section{RESULTATS ET DISCUSSION}

\subsection{Fluage et essais dynamiques}

Les observations ont montré que le mécanisme régissant la déformation à l'échelle de la microstructure est le cisaillement des précipités par des configurations faisant intervenir des superdéfauts d'empilement intrinsèques (S-ISF) et extrinsèques (S-ESF) [2 - 5]. Les faits suivants ont été mis en évidence :

- La nature des superdéfauts d'empilement dépend de la nature du test effectué : La majorité des défauts est intrinsèque (S-ISFs) en fluage traction (fig. 1) tandis qu'en compression dynamique (fig. 2), ceux-ci sont en majorité extrinsèques (S-ESFs). Il y a un mélange de S-ESFs et de S-ISFs en fluage compression (Fig. 3) et en traction dynamique (Fig. 4). Deux plans sont affectés par le cisaillement pour la S-ESF (Fig. 5) et un seul l'est pour la S-ISF (Fig. 6).

- Les configurations de cisaillement font intervenir des dislocations de matrice $a / 2<110\rangle$ dissociées et il a été prouvé qu'une seule dislocation de matrice pouvait initier le cisaillement (Fig. 2). Il existe à l'interface $\gamma /$ $\gamma^{\prime}$ des dislocations de Shockley différentes de celles qui cisaillent le précipité (Fig. 2). Une analyse fine de la structure de ces dislocations a été obtenue en haute résolution (Fig. 5).

- De très fins couloirs de matrice sont présents au sein des précipités (Fig. 1) et une montée de type « pipe diffusion » est active au sein de ceux-ci.

Ces faits nous ont conduits à remettre en question le mécanisme de cisaillement proposé par Kear il y a une vingtaine d'années [6]. La conjonction des observations en champ faible et en champ clair nous a permis de proposer un mécanisme original de cisaillement des précipités qui a une réelle signification physique à partir d'une simple dislocation de matrice $a / 2<110>$ dissociée : Il a ainsi pu être rendu compte de l'asymétrie traction/compression que nous avons observée dans la direction $<001>$.

Cette asymétrie peut être expliquée si l'on tient compte de deux effets dont la résultante peut conduire à la présence de S-ESF, S-ISF ou bien du mélange des deux :

- une barrière d'énergie au franchissement de l'interface par une partielle de Shockley.

- l'effet de la force résolue (venant de la contrainte appliquée) sur la largeur de dissociation de la dislocation de matrice. Il a été montré que pour l'orientation $<001\rangle$, une contrainte de compression augmente la largeur de dissociation tandis qu'une contrainte de traction la diminue.

Le modèle est décrit en Fig. 7 pour le cas extrinsèque et en Fig. 8 pour l'intrinsèque:

En compression dynamique, la contrainte appliquée augmente la largeur de dissociation ce qui conduit à la formation de S-ESF tandis qu'en fluage traction, c'est l'inverse, la contrainte appliquée diminue la largeur de dissociation conduisant à la formation de S-ISF. Une balance entre les deux effets conduit aux mélanges de défauts observés dans les deux autres cas.

Un tel mécanisme permet d'expliquer la dépendance vis-à-vis de la température des modes de cisaillement des précipités en fluage primaire.

Il permet également de comprendre la dissymétrie observée à l'échelle macroscopique en début de fluage primaire à température intermédiaire : résistance plus grande en traction qu'en compression pour une même déformation plastique. En effet, il est nécessaire en traction de recombiner les dislocations et d'inverser les partielles pour autoriser le cisaillement des précipités.

\subsection{Fatigue oligocyclique}

Les observations ont été effectuées sur l'alliage AM1 pour des déformations totales comprises entre $0,6 \%$ à $2,2 \%$ et un nombre de cycles variant de la simple traction $(\mathrm{N}=1)$ jusqu'à la rupture $\left(\mathrm{N} \approx 10^{4}\right)$.

Après une simple traction $\left(\Delta \varepsilon_{\mathrm{t}}=1,25 \%\right)$, la déformation est anisotrope et s'accumule aux interfaces perpendiculaires à l'axe de sollicitation (couloirs horizontaux) et forme des réseaux qui réduisent l'écart 
paramétrique (Fig. 9). Si l'on augmente le nombre de cycles pour un même taux de déformation totale (25 cycles), la déformation reste anisotrope mais commence à s'étendre dans les autres couloirs (Fig. 10). Les réseaux de dislocations sont toujours présents et réduisent l'écart paramétrique. Pour un nombre de cycles encore plus important, la déformation devient homogène c'est à dire que la densité de dislocations dans les couloirs perpendiculaires et parallèles à la contrainte est du même ordre de grandeur. Dans ce cas, certaines dislocations appartenant aux réseaux plaqués aux interfaces ne réduisent plus l'écart paramétrique mais le renforcent. A plus faible déformation totale imposée $\left(\Delta \varepsilon_{\mathrm{t}}=0,63 \%, 200\right.$ cycles $)$, la déformation est anisotrope et la microstructure présente une coalescence de type $\mathrm{N}$ comme en fluage traction (Fig. 11). Pour une plus forte déformation totale imposée $\left(\Delta \varepsilon_{1}=2,2 \%, 200\right.$ cycles $)$, la déformation est homogène et le mécanisme de cisaillement par paires devient significatif (Fig. 12).

Toutes ces observations nous ont permis de dresser, pour la première fois en fatigue oligocyclique, une carte décrivant l'évolution des microstructures en fonction du taux de déformation imposée et du nombre de cycles ainsi qu'une interprétation du comportement de l'alliage. Cette carte est présentée en Fig. 13.

Pour de fortes déformations totales imposées, la contrainte imposée étant beaucoup plus élevée active de façon très significative le cisaillement par paires. Cette augmentation de contrainte favorise par ailleurs les phénomènes de migration atomique [7] et la montée des dislocations [8] provoquant une rapide homogénéisation de la microstructure. A plus faible déformation totale imposée, l'écart paramétrique introduit une anisotropie de contraintes internes qui inhibe la déformation dans les couloirs verticaux. Les contraintes dues à l'écart paramétrique favorisent le mouvement des dislocations qui relaxent cet écart provoquant ainsi une anisotropie de la déformation. Si l'on augmente le nombre de cycles, la diffusion devient effective et la présence de gradients élastiques entre les interfaces dus aux dislocations provoque une coalescence de type $\mathrm{N}[9]$.

\section{CONCLUSIONS}

L'étude des microstructures de déformation localisées aux interfaces $\gamma / \gamma$ dans les superalliages base nickel nous a permis de remonter aux mécanismes élémentaires régissant cette déformation.

En particulier, le couplage des techniques de microscopie électronique en transmission en champ faible et en haute résolution, permettant une étude « tridimensionnelle » des dislocations situées aux interfaces, nous a amenés à proposer un mécanisme original de cisaillement des précipités en fluage primaire. De la même façon, l'étude fine des réseaux de dislocations bloqués aux interfaces $\gamma / \gamma^{\prime}$ a permis d'obtenir pour la première fois une description général du comportement en fatigue oligocyclique à $950^{\circ} \mathrm{C}$.

\section{Remerciements}

Les auteurs remercient la Société SNECMA et l'ONERA pour leur avoir fournis respectivement l'AM1 et l'AM3. Ils remercient également le CPR «SSSM » pour le financement des études. Ils remercient également Monsieur le Professeur J. Philibert (LMS, Orsay) pour d'intéressantes discussions.

\section{Références}

[1] Cockayne D.J.H., Ray, I.L.F. et Whelan M.J., Phil. Mag. 20 (1969), 1265.

[2] Condat M. et Décamps B., Scripta Metall., 21 (1987), 607.

[3] Décamps B., Morton A.J. et Condat M., Phil. Mag. A, 64 (1991), 3, 641.

[4] Décamps B. et Morton A.J., Phil. Mag. A, 68 (1993), 1129.

[5] Décamps B., Pénisson J.M., Condat M., Guétaz L. et Morton A.J., Scripta Metall., 30 (1994), 1425.

[6] Kear B.H. et Oblak J.M., J. Phys. Paris, 35 (1974), C7-35.

[7] Philibert J. , Atom movement, diffusion and mass transport in solids,Ed. de Phys. 1991.

[8] Hirth J.P. and Lothe J., Theory of dislocations, 2nd Ed., Wiley-Interscience Pub., 1982, P. 558.

[9] Véron M., Thèse de l'INPG, Grenoble (1995). 


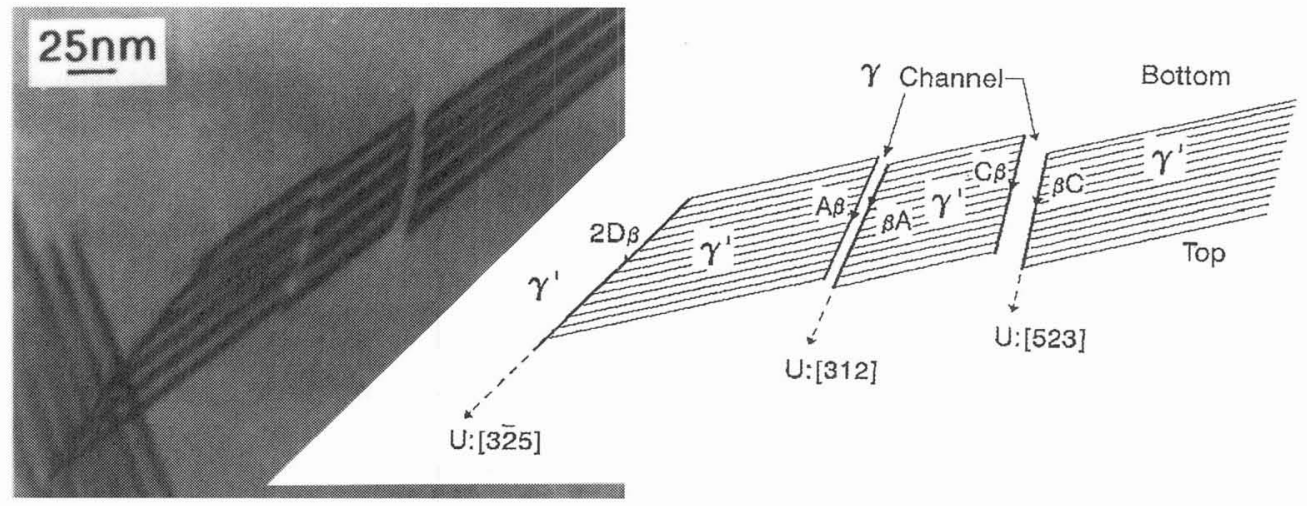

Fig. 1 : Alliage $\mathrm{AM} 3$ déformé en fluage traction à $760^{\circ} \mathrm{C}$; Défaut $(\mathrm{B}=(594), \mathrm{g}=1 \overline{1} 1)$ et sa représentation schématique.

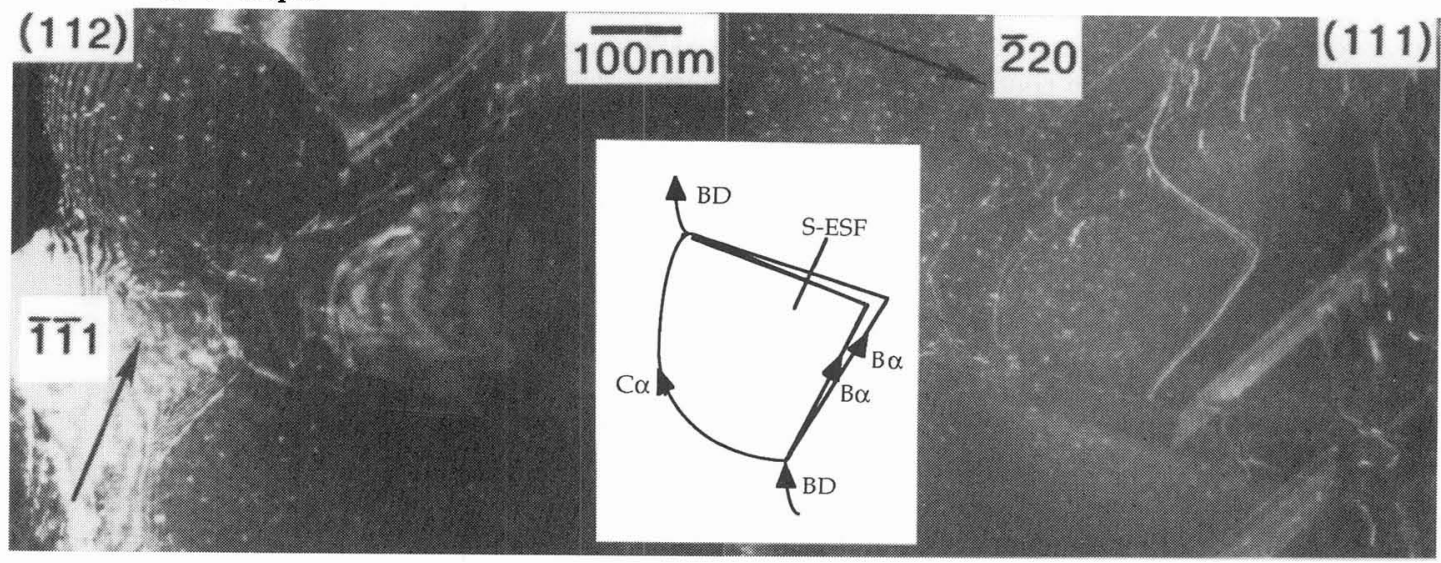

Fig. 2: Alliage AM1 déformé en compression dynamique ; Défaut et sa représentation schématique.
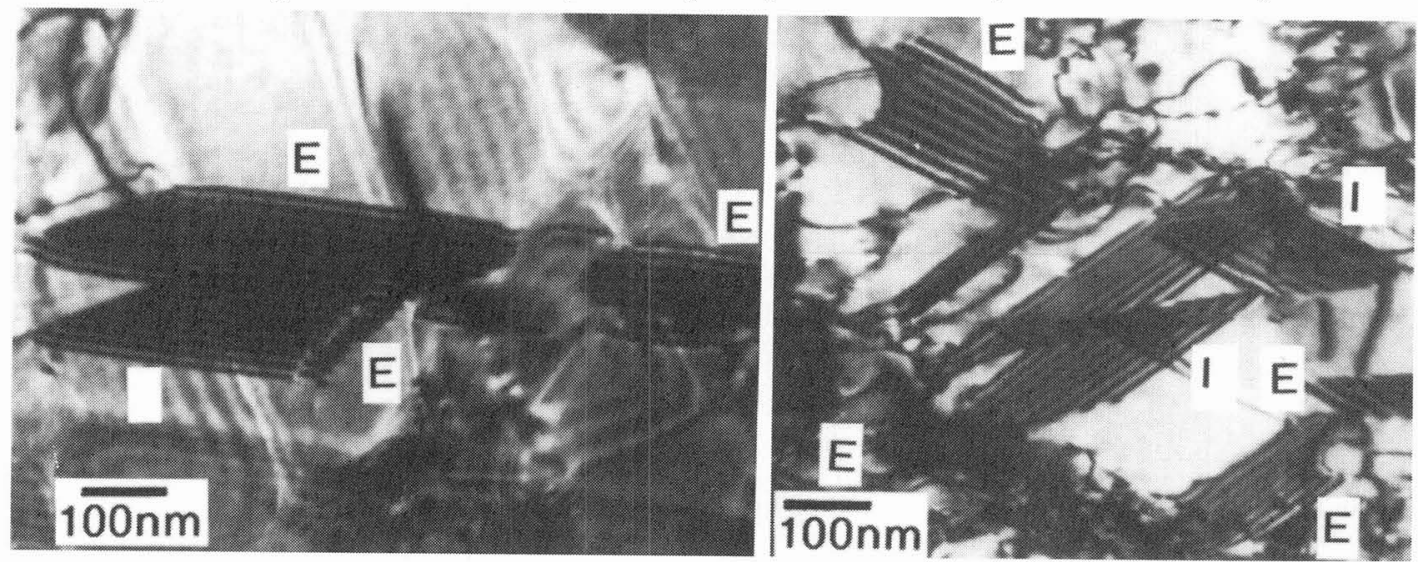

Fig. 3 : Alliage AM1 déformé en fluage compression Fig. 4 : Alliage AM1 déformé en traction dynamique à $810^{\circ} \mathrm{C}(\mathrm{E}=\mathrm{S}$-ESF; $\mathrm{I}=\mathrm{S}-\mathrm{ISF})$. à $950^{\circ} \mathrm{C}(\mathrm{E}=\mathrm{S}$-ESF; I = S-ISF). 


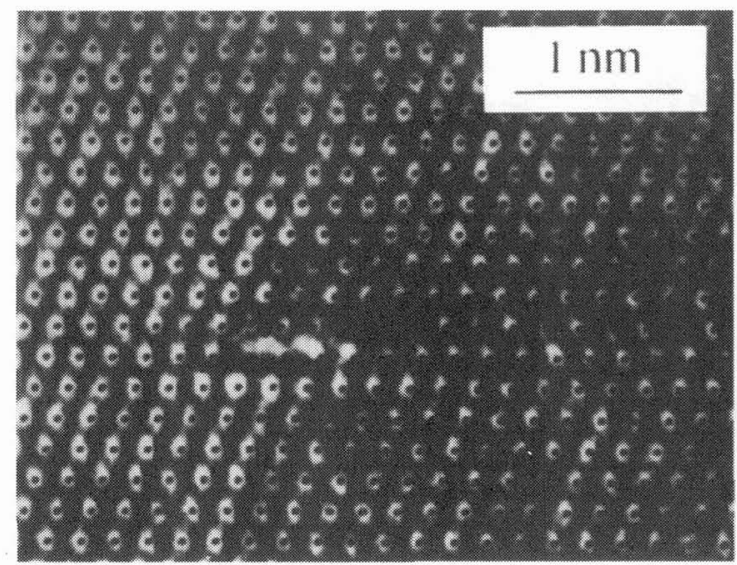

Fig. 5 : Micrographie en haute résolution d'une S-ESF.

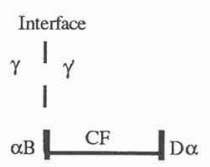

(a)

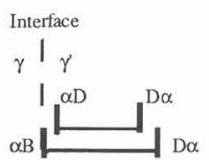

(b) (a) : franchissement de l'interface $\gamma / \gamma^{\prime}$ par une dislocation dissociée DB sans inversion des partielles. (b) : nucléation d'une dislocation de Shockley $\alpha \mathrm{D}$ sur un plan adjacent au dessus de la faute complexe sur la partielle de queue conduisant à la formation de la S-ESF.

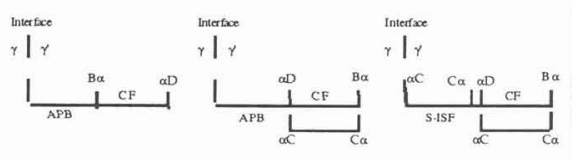
(a) (b) (a)

(a) : franchissement de l'interface $\gamma / \gamma^{\prime}$ par une dislocation dissociée $\mathrm{BD}$ avec inversion des partielles.

(b) : dissociation des partielles de Shockley bordant la faute complexe sur 2 plans adjacents, dissociation équivalente à celle des CFC pour les fautes extrinsèques. (c) : nucléation d'une dislocation de Shockley $\mathrm{C} \alpha$ au sein de l'APB sur la partielle de queue conduisant à la formation de la S-ISF et d'un dipole de Shockley $\mathrm{C} \alpha / \alpha \mathrm{C}$ sur des plans adjacents.

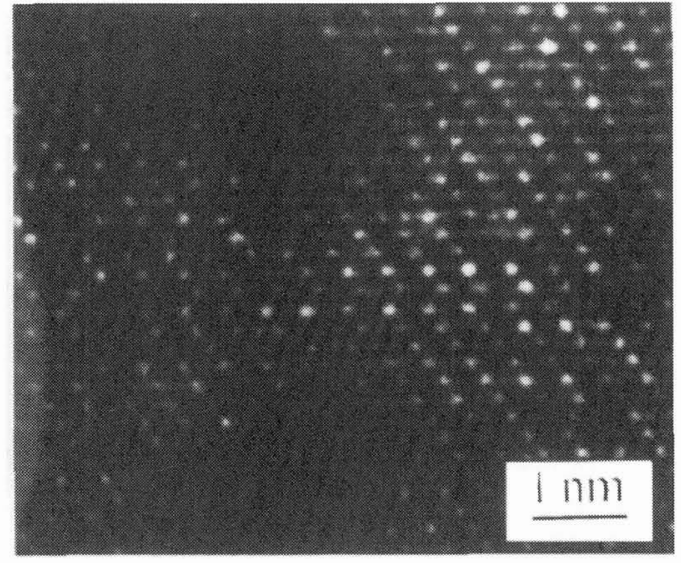

Fig. 6 : Micrographie en haute résolution d'une S.ISF
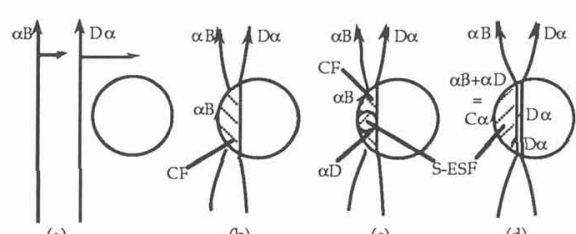

(c)

Schéma équivalent mais dans la même projection que le champ faible (projection (111).

Fig. 7 : Mécanisme de formation d'une SESF à partir d'une dislocation unique de matrice dissociée.
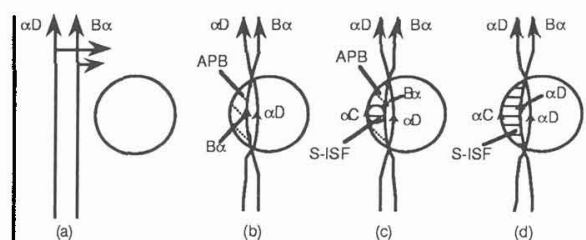

Schéma équivalent mais dans la même projection que le champ faible (projection (111).

Fig. 8 : Mécanisme de formation d'une SISF à partir d'une dislocation unique de matrice dissociée. 


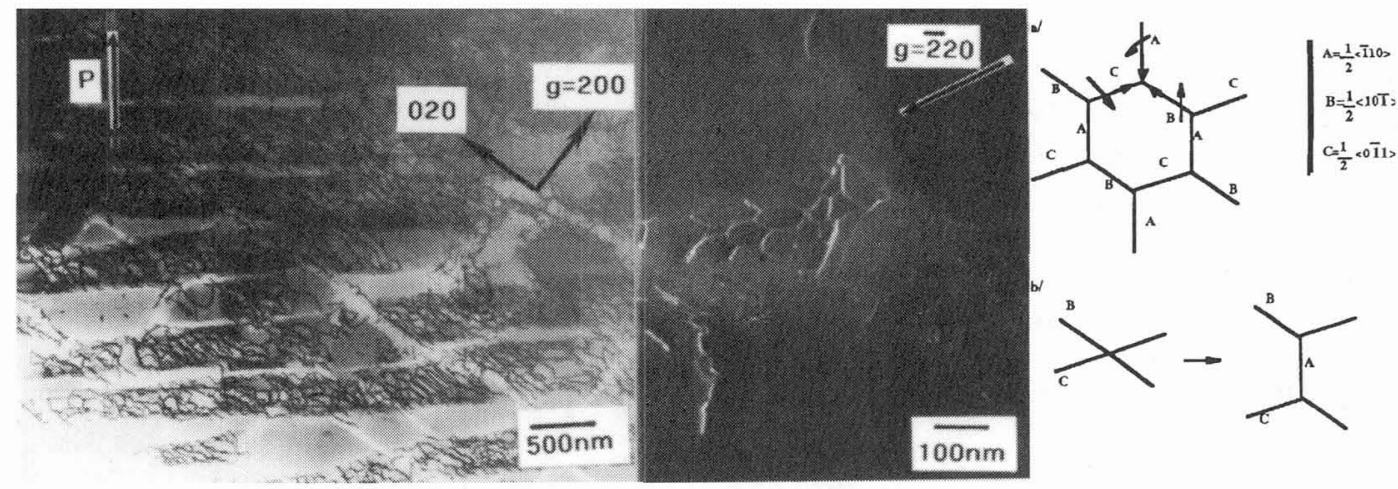

Fig. $9: \Delta \varepsilon_{\mathrm{t}}=1,25 \%, \mathrm{~N}=1 . \mathrm{P}=$ projection de l'axe de sollicitation. Présence de réseaux aux interfaces avec leur indexation (Vecteurs de Burgers en a) et proposition d'intersection de deux systèmes en b).

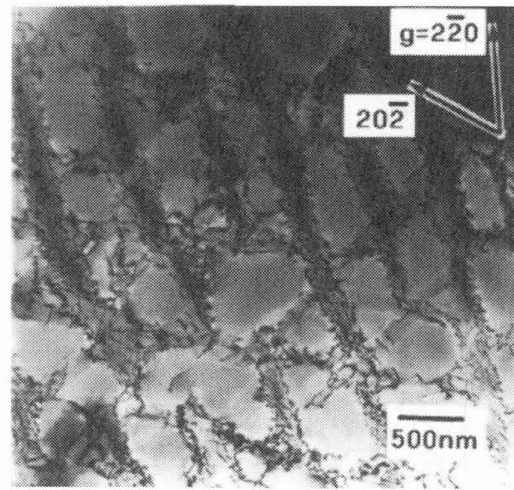

Fig. $10: \Delta \varepsilon_{\mathrm{t}}=1,25 \%$, $\mathrm{N}=25$ cycles.

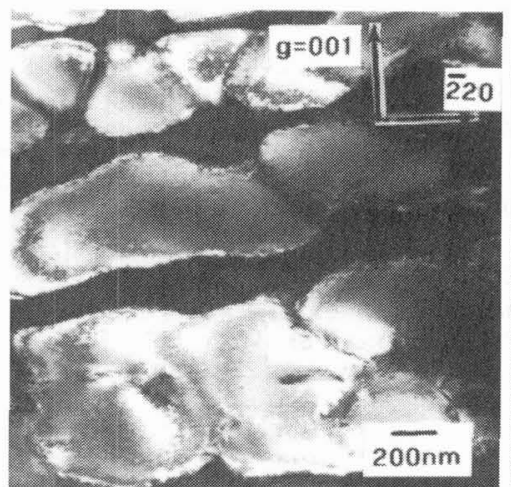

Fig. $11: \Delta \varepsilon_{\mathrm{t}}=0,63 \%$, $\mathrm{N}=200$ cycles.

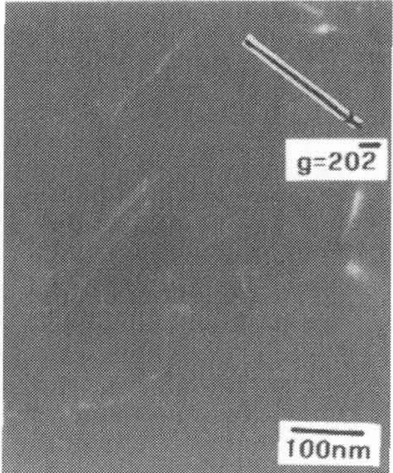

Fig. $12: \Delta \varepsilon_{\mathrm{t}}=2,2 \%$, $\mathrm{N}=200$ cycles

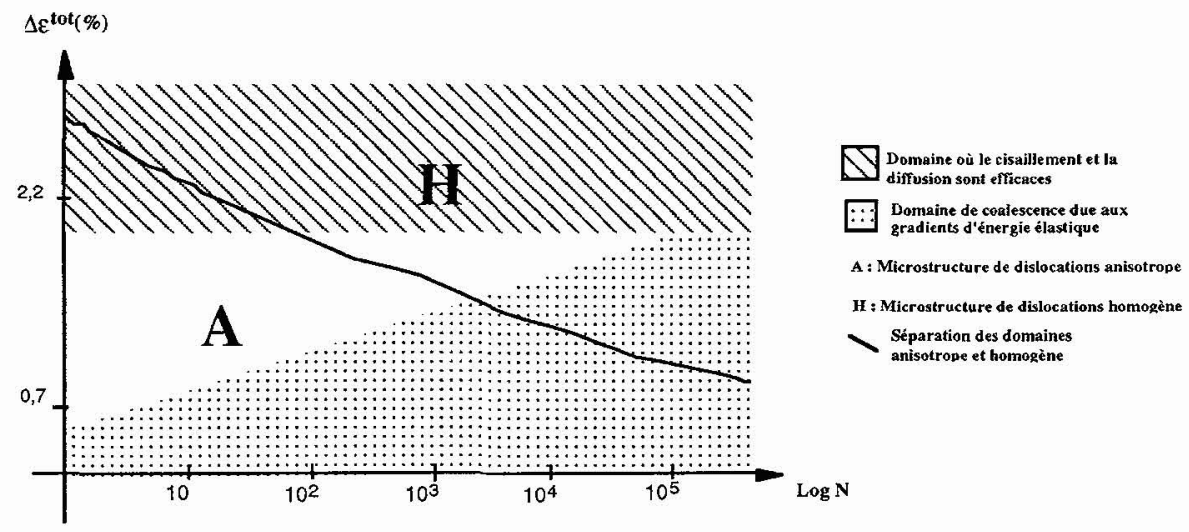

Fig. 13 : Carte de déformation du superalliage $A M 1$ en fatigue oligocyclique à $R_{\varepsilon}=0$ à $950^{\circ} \mathrm{C}$. 